\title{
Stent-grafts in the management of life-threatening hemorrhage following inadvertent femoral catheterization in high-risk patients: report of two cases and review of the literature
}

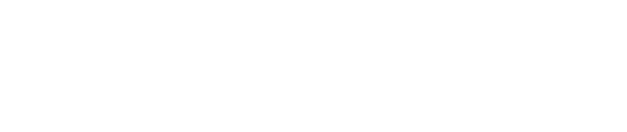

\author{
Abdullah Al Wahbi \\ Division of Vascular Surgery, \\ Department of Surgery, King \\ Abdulaziz Medical City, Riyadh, \\ Saudi Arabia
}

Correspondence: Abdullah AI Wahbi Division of Vascular Surgery, Department of Surgery, King Abdulaziz Medical City, PO Box 22490, Riyadh II426,

Saudi Arabia

Tel +966 2520088 ext |4 |37 + |4| I7

Fax +966252005 I

Email alwahbil@hotmail.com

\begin{abstract}
Bleeding related to inadvertent femoral catheter insertion is an infrequent but morbid complication. We report two cases of acute life-threatening bleeding after attempts at femoral vein catheterization. Both patients were morbidly obese, had multiple comorbidities, and were at high risk for surgical intervention. Aggressive resuscitation failed to stabilize the patients. Emergency digital subtraction angiography diagnosed the site of the femoral artery bleeding. In one patient, the site of injury was the right profunda femoris artery. Both bleeding sites were controlled successfully with endovascular covered stent placement, without complication. Although prior publications have reported the concept of endovascular repair in femoral artery injuries, to the best of the author's knowledge, covering the profunda femoris artery with no complications has not been described previously. In critically ill patients with life-threatening hemorrhage following inadvertent femoral catheterization, management with endovascular covered stents is safe and effective.
\end{abstract}

Keywords: critical care, central venous catheterization, iatrogenic complications

\section{Introduction}

Femoral vein catheterization is often carried out during resuscitation and in critical care units to provide venous access and nutritional support or to manage perioperative fluids. It is usually achieved via a blind approach, localizing the femoral pulse manually using external landmarks. However, this approach can be challenging in patients who are obese or have severe shock, as the femoral pulse could be absent, weak, or too deep to palpate. Inadvertent femoral catheter insertion can cause hemorrhage, pseudoaneurysm, arteriovenous fistulation, arterial dissection, or neurological injury. ${ }^{1,2}$ Operative repair has been the traditional treatment. However, for active bleeding in obese patients with abnormal coagulation and a high risk to conventional anesthesia, endovascular treatment could be seen as a safe and effective therapeutic alternative. ${ }^{1-3}$

\section{Case I}

A 66-year-old female who was morbidly obese and known to have diabetes mellitus, hypertension, and renal impairment was admitted to the intensive care unit (ICU) with acute biliary pancreatitis and active rectal bleeding due to colonic polyps. After admission, she developed massive per-rectum bleeding with hypovolemic shock. The patient's pulse was weak and her blood pressure could not be measured accurately using a pressure cuff. Her peripheral veins were difficult to cannulate because of her 
obesity and the hypovolemic shock. During resuscitation, several attempts to insert a large-bore catheter (8.5 Fr high flow fluid administration set) in the right femoral vein failed, and the procedure was aborted. A firm pressure dressing was applied to the puncture site. A left jugular catheter was successfully inserted, and fluid and blood resuscitation started. The patient was stabilized; however, she continued to be in a critical condition and developed coagulopathy. A few hours later, the patient developed a groin hematoma, which was rapidly progressing in size. An urgent color duplex ultrasound scan showed an $8 \mathrm{~cm} \times 10 \mathrm{~cm}$ femoral pseudoaneurysm that was actively bleeding. Laboratory studies showed a fall in hemoglobin from 9 to $5 \mathrm{~g} / \mathrm{dL}$. The patient developed hypovolemic shock, and resuscitation was difficult. The right groin hematoma continued to increase in size. As the patient was at high risk for conventional anesthesia due to her comorbidities, endovascular therapy was planned instead of surgery. Under local anesthesia, an angiography was performed via a left femoral approach. There was evidence of active bleeding from the right superficial femoral artery just beyond the common femoral bifurcation (Figure 1A). An $8 \mathrm{~mm} \times 40 \mathrm{~mm}$ self-expanding polytetrafluoroethylene (PTFE)-covered stent (Fluency; C.R. Bard, Inc., Murray Hill, NJ, USA) was deployed, and bleeding was successfully controlled (Figure 1B). No complications related to the procedure were observed. The patient went back to the ICU when she was stabilized.

\section{Case 2}

A 60-year-old female with morbid obesity was admitted to the ICU with septic shock due to peritonitis. She was known to have end-stage renal disease on peritoneal dialysis, diabetes, and hypertension. Several attempts to insert a right femoral dialysis access failed. The patient developed a groin hematoma

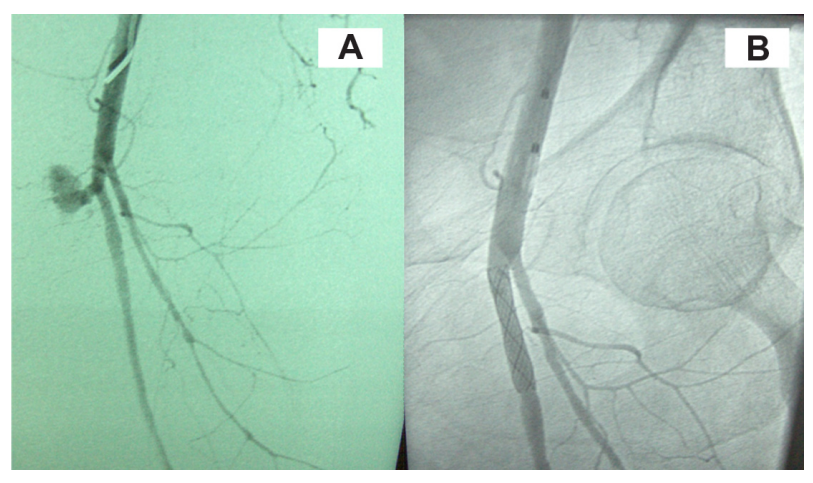

Figure I Case I. A) Extravasation from the superficial femoral artery just beyond the common femoral bifurcation. B) Successful control of the bleeding after stent deployment. with a drop in her hemoglobin. An ultrasound confirmed active bleeding from the right femoral artery, and the patient was prepared for surgical repair. However, before transfer to the operating room, the patient collapsed with hypotension and an increase in the right groin hematoma. Her hemoglobin dropped from 8 to $4 \mathrm{~g} / \mathrm{dL}$ and she developed coagulopathy. She was resuscitated and transferred to the angiography suite where an angiogram confirmed the site of active bleeding to be from the deep femoral artery (Figure 2A). An $8 \mathrm{~mm} \times 40 \mathrm{~mm}$ self-expanding PTFE-covered stent (Fluency; C.R. Bard, Inc.) was deployed in the common femoral artery, extending to the superficial femoral artery and occluding the deep femoral artery (Figure 2B). An angiogram showed patent superficial femoral, popliteal, and distal vessels. No complications related to the procedure were observed.

\section{Discussion}

Central venous catheter (CVC) placement is an essential part of clinical management of many conditions in hospitalized patients. CVCs are inserted for several reasons, including hemodynamic monitoring, fluid replacement, hemodialysis, total parenteral nutrition, management of perioperative fluids, and delivery of blood products and drugs such as vasopressors, chemotherapy, and antibiotics. ${ }^{4}$ In addition, they are a vital tool for critically ill patients who require aggressive fluid management and intensive care. ${ }^{5,6}$ The common femoral vein is a common access site for CVC placement. Their insertion is usually achieved via a blind, external landmark-guided technique, through manual localization of the femoral artery. Iatrogenic arterial injury at the puncture site is a potential complication of CVC placement. It can present acutely with life-threatening blood loss or can insidiously lead to a pseudoaneurysm formation. ${ }^{7}$ Femoral catheterization has a higher incidence of mechanical complications than

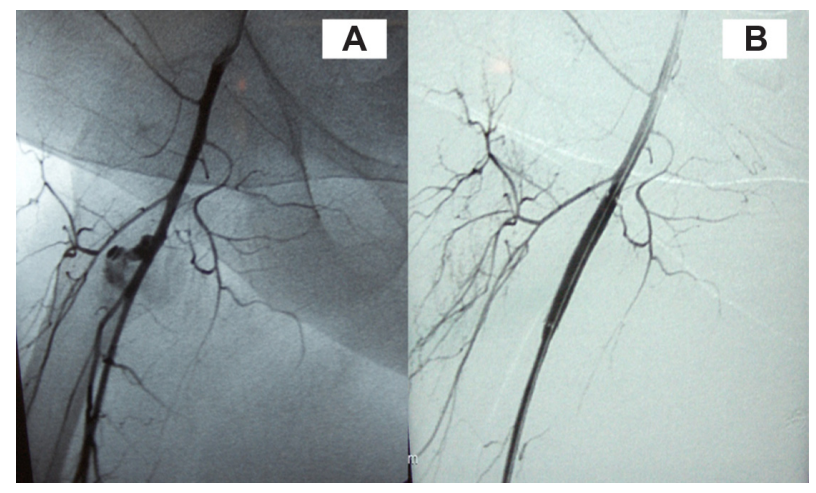

Figure 2 Case 2. A) Active extravasation from the deep femoral artery just beyond the common femoral bifurcation. B) Successful control of the bleeding after stent deployment. 
the subclavian or internal jugular access, ${ }^{8}$ with femoral or retroperitoneal hematoma the most frequent major complication, occurring in up to $1.3 \%$ of cases. ${ }^{1}$ Other complications include hematoma, pseudoaneurysm, arteriovenous fistula, arterial dissection, thrombosis, distal embolization, nerve damage, abscess, and lymphocele. ${ }^{2}$ There are possible factors that influence the risk of these complications. They include a faulty puncture technique, ie, low or high puncture sites, either puncture above the inguinal ligament or puncture of the superficial femoral artery or deep femoral artery; ${ }^{9}$ poor postprocedural compression; length of procedure; multiple attempts; larger devices; and more aggressive anticoagulation during and following the procedure. ${ }^{10,11}$ Experience has been reported to be another factor. ${ }^{12}$ Patient-specific risk factors include advanced age, female gender, obesity, coagulopathy, peripheral arterial disease with heavy arterial calcification of the femoral and iliac arteries, hypertension, hemodialysis, hostile groin (excessive scarring following surgery or repeated angiography, or graft material), high division of femoral artery, and nonflexible hip. ${ }^{13,14}$ The presence of pain or swelling in the groin after catheterization is the most common presentation of a femoral artery injury. Hemodynamic instability and a fall in hemoglobin suggest a life-threatening rupture, which may be occult if retroperitoneal. ${ }^{10}$

Reports of the advantages of ultrasonography over the anatomic landmark method support the findings of risk reduction and improved cannulation success for all access sites. ${ }^{9,15}$ The diagnostic examination of choice is duplex ultrasound, which can detect iatrogenic injuries of the femoral artery at the groin with $94 \%$ and $97 \%$ reported sensitivity and specificity, respectively. ${ }^{16}$ Management of access site complications may include conservative therapy, percutaneous intervention, and/or vascular surgery. ${ }^{17}$ Ultrasound-guided compression can be used in minor injuries and stable patients; however, it has major limitations, including patient intolerance for those with compression pain, obese patients, and those who are receiving anticoagulants. ${ }^{16,18}$ Percutaneous ultrasound-guided thrombin injection is currently the method of choice in many centers for femoral pseudoaneurysms, with a $91 \%$ to $100 \%$ success rate in large series. ${ }^{9}$ In obese patients with coagulopathy and hemodynamic instability, these methods are less successful in controlling active bleeding, and urgent surgical vascular repair is indicated. ${ }^{5}$ The success of surgical repair of iatrogenic femoral access lesions is nearly $100 \%$. However, it may be complicated by several factors, such as anatomical accessibility if the vascular lesion lies deep in the pelvis, and patients' comorbid conditions, which may augment related morbidity to $25 \%$ and mortality to $3.5 \% .^{5}$ In addition, the existing hematoma and local tissue injuries frequently found in such iatrogenically traumatized areas predispose to poor wound healing and postoperative infection, especially if interposition of a synthetic graft is needed.

Endovascular treatment with covered self-expanding stent-grafts has been reported as an effective method in the management of acute arterial bleeding. ${ }^{8,11,14,15}$ It is performed under local anesthesia, which avoids the need for general or locoregional anesthesia in unstable, high-risk patients. There are several limitations to this procedure, including cost, unfavorable vessel anatomy, and the potential fracture of the stent at a site of mobility near the hip. The risk of covering the deep femoral artery is considered a limitation of the procedure, particularly in patients with severe peripheral arterial disease. ${ }^{11,13}$ However, in life-threatening situations where an endovascular approach is the only option, saving the patient's life is a priority over limb perfusion. In our case, the site of injury was the deep femoral artery, which was covered with no effect on the lower limb perfusion, as the superficial femoral artery and distal vessels were patent.

Although there are no long-term studies available at this point comparing different management methods of femoral artery inadvertent injuries, an endovascular approach, with its minimally invasive nature, and short and relatively painless recovery period, appears to be a significant and effective alternative to surgical treatment, especially in high-risk patients.

\section{Conclusion}

This study suggests that endovascular therapy with stent-graft is a safe, effective, and life-saving procedure in the management of active life-threatening femoral artery bleeding when traditional open surgery is contraindicated.

\section{Disclosure}

The author reports no conflicts of interest in this work.

\section{References}

1. Merrer J, de Jonghe B, Golliot F, et al. Controlled trial catheterization in critically ill patients: a randomized complications of femoral and subclavian venous. JAMA. 2001;286(6):700-707.

2. Pikwer A, Acosta S, Kölbel T, Malina M, Sonesson B, Akeson J. Management of inadvertent arterial catheterisation associated with central venous access procedures. Eur J Vasc Endovasc Surg. 2009;38(6):707-714

3. Gabriel M, Pawlaczyk K, Waliszewski K, Krasiński Z, Majewski W. Location of femoral artery puncture site and the risk of postcatheterization pseudoaneurysm formation. Int J Cardiol. 2007;120(2):167-171

4. Tercan F, Ozkan U, Oguzkurt L. US-guided placement of central vein catheters in patients with disorders of hemostasis. Eur J Radiology. 2008; 65:253-256

5. Tana PL, Gibson M. Central venous catheters: the role of radiology. Clin Radiol. 2006;61:13-22. 
6. Kapadia S, Parakh R, Grover T, Agarwal S, Yadav A. Endovascular covered stent for management of arterial pseudoaneurysms after central venous access. J Cardiothorac Vasc Anesth. 2007;21(1):99-102.

7. Arat A, Turkbey B, Cil BE, Canyigit M, Cekirge S. Emergent treatment of an Iatrogenic arterial injury at femoral puncture site With Symbiot ${ }^{\mathbb{B}}$ self-expanding PTFE-covered coronary stent-graft. Australas Radiol. 2007;51:B331-B333.

8. Kusminsky RE. Complications of central venous catheterization. J Am Coll Surg. 2007;204(4):681-696.

9. Webber GW, Jang J, Gustavson S, Olin JW. Contemporary management of postcatheterization pseudoaneurysms. Circulation. 2007;115:2666-2674.

10. Ahmad F, Turnery SA, Torrie P, Gibson M. Iatrogenic femoral artery pseudoaneurysms. A review of current methods of diagnosis and treatment. Clin Radiol. 2008;63:1310-1316.

11. Samal AK, White CJ. Percutaneous management of access site complications. Catheter Cardiovasc Interv. 2002;57:12-23.

12. Balls A, LoVecchio F, Stapczynski S. Central line emergency access registry: a multi-center study to determine resident competency with central venous catheter insertion. Ann Emerg Med. 2007;50:S115.
13. Tsetis D. Endovascular treatment of complications of femoral arterial access. Cardiovasc Intervent Radiol. 2010;33:457-468.

14. Heis HA, Bani-Hani KE, Elheis MA, Yaghan RJ, Bani-Hani BK. Postcatheterization femoral artery pseudoaneurysms: therapeutic options. A case-controlled study. Int J Surg. 2008;6:214-219.

15. Pikwer A, Acosta S, Kölbel T, Malina M, Sonesson B, Akeson J. Management of inadvertent arterial catheterisation associated with central venous access procedures. Eur J Vasc Endovasc Surg. 2009;38:707-714.

16. Demirbas O, Batyraliev T, Eksi Z, Pershukov I. Femoral pseudoaneurysm due to diagnostic or interventional angiographic procedures. Angiology. 2005;56:553.

17. Guilbert MC, Elkouri S, Bracco D, et al. Arterial trauma during central venous catheter insertion: case series, review and proposed algorithm. J Vasc Surg. 2008;48(4):918-925.

18. Hill G, Albayati S, Thomson I, Macfarlane J, Dutton S, van Rij AM. Iatrogenic pseudoaneurysms: optimal management and treatment options. J Vasc Ultrasound. 2007;31(3):131-135.
Vascular Health and Risk Management

\section{Publish your work in this journal}

Vascular Health and Risk Management is an international, peerreviewed journal of therapeutics and risk management, focusing on concise rapid reporting of clinical studies on the processes involved in the maintenance of vascular health; the monitoring, prevention and treatment of vascular disease and its sequelae; and the involvement of

\section{Dovepress}

metabolic disorders, particularly diabetes. This journal is indexed on PubMed Central and MedLine. The manuscript management system is completely online and includes a very quick and fair peer-review system, which is all easy to use. Visit http://www.dovepress.com/ testimonials.php to read real quotes from published authors. 